Review

\title{
In vivo antioxidant activity of phenolic compounds: Facts and gaps
}

\author{
Natália Martins ${ }^{\text {a, b }}$, Lillian Barros ${ }^{\text {a }}$, Isabel C.F.R. Ferreira ${ }^{\text {a, * }}$ \\ ${ }^{a}$ Mountain Research Centre (CIMO), ESA, Polytechnic Institute of Bragança, Campus de Santa Apolónia, Apartado 1172, 5301-855 Bragança, Portugal \\ ${ }^{\mathrm{b}}$ CEB, Centre of Biological Engineering, LIBRO-Laboratório de Investigação em Biofilmes Rosário Oliveira, University of Minho, 4710-057 Braga, Portugal
}

\section{A R T I C L E I N F O}

\section{Article history:}

Received 29 August 2015

Received in revised form 17 November 2015

Accepted 23 November 2015

Available online 2 December 2015

\section{Keywords:}

Aging-related diseases

Antioxidant activity

In vivo studies

Phenolic extracts/compounds

Bioavailability

\begin{abstract}
A B S T R A C T
Background: Numerous diseases have been related with free radicals overproduction and oxidative stress. Botanical preparations possess a multitude of bioactive properties, including antioxidant potential, which has been mainly related with the presence of phenolic compounds. However, the mechanisms of action of these phytochemicals, in vivo effects, bioavailability and bio-efficacy still need research. Scope and approach: The present report aims to provide a critical review on the aspects related with the in vivo antioxidant activity of phenolic extracts and compounds from plant origin.

Key findings: Biological functions beyond the human metabolism were discussed, comparing in vivo vs. in vitro studies, as also focusing the conditioning factors for phenolic compounds bioavailability and bioefficacy. Furthermore, an upcoming perspective about the use of phytochemicals as life expectancy promoters and anti-aging factors in human individuals was provided.

Conclusions: Overall, and despite all of those advances, the study of the biological potential of numerous natural matrices still remains a hot topic among the scientific community. In fact, the available knowledge about the responsible phytochemicals for the biological potential, their mechanisms of action, the establishment of therapeutic and prophylactic doses, and even the occurrence of biochemical interrelations, is considerable scarce.
\end{abstract}

(C) 2015 Elsevier Ltd. All rights reserved.

\section{Introduction}

In the last years, oxidative stress-related diseases/disorders have gained a special attention. Metabolic, neurodegenerative, cardiovascular, mitochondrial diseases and even cancer, are among the most frequent (Chaturvedi \& Beal, 2013; Halliwell, 2012; Singh, Sharad, \& Kapur, 2004). Numerous studies have been investigating the underlying triggering factors, in order to understand the mechanisms of action of free radicals, as well as to discover effective substances towards preventing and even reversing the occurrence of oxidative damages (Espín, García-Conesa, \& TomásBarberán, 2007; Fernandez-Panchon, Villano, Troncoso, \& GarciaParrilla, 2008).

Antioxidants, both from natural and synthetic sources, have proved to be highly effective to control the magnitude of free radicals production, to prevent its undesirable effects, as well as to support the organism antioxidant and detoxifying mechanisms (Holst \& Williamson, 2008; Kapravelou et al., 2015; Valko et al.,

\footnotetext{
* Corresponding author.

E-mail address: iferreira@ipb.pt (I.C.F.R. Ferreira).
}

2007; Yeh \& Yen, 2006).

Phenolic compounds have shown promising antioxidant properties, with its potential being directly related with the type of solvent used in the extraction, but also with plant origin, growing conditions, harvesting time, and storage conditions (Avello, Pastene, Bustos, Bittner, \& Becerra, 2013; Taârit, Msaada, Hosni, \& Marzouk, 2012; Trabelsi et al., 2012). The study of the antioxidant potential of phenolic extracts derived from plant species is one of the hot topics among the scientific community; however, in vitro studies are the most common (Dai \& Mumper, 2010; Larrosa, García-Conesa, Espín, \& Tomás-Barberán, 2010; Rubió, Motilva, \& Romero, 2013). Nevertheless, these studies do not consider biochemical, metabolic and other physiological parameters (Devasagayam et al., 2004; Espín et al., 2007; Fernandez-Panchon et al., 2008). In vivo studies have been mainly performed in eukaryotic cells, mice, fishes, guinea-pigs and rabbits, but studies involving human clinical trials remain scarce (Fernandez-Panchon et al., 2008; Goodman, Bostick, Kucuk, \& Jones, 2011; Rubió et al., 2013). Several biological and physiological variables can be accessed and measured by those studies, including the metabolism of the tested matrices, as well as their bioavailability (Espín et al., 2007; Holst \& Williamson, 2008). 
Not only natural but also synthetic antioxidants suffer numerous biochemical reactions along ingestion, digestion and absorption by the organisms. Therefore, and despite the current advances, the effective bioavailability of the different antioxidants is not clearly defined: while many of them are ingested on its active form, others need to be metabolized to be biologically active, or even become inactive; furthermore, the co-ingestion of other nutrients, as also several endogenous factors, and the inter and intraindividual variations, affect their bioavailability in relation to the ingested dose (Espín et al., 2007; Heim, Tagliaferro, \& Bobilya, 2002; Holst \& Williamson, 2008). This fact explains why some plant species and even isolated compounds do not evidence positive effect through in vitro studies, but a strong antioxidant potential is observed when in vivo studies are carried out, and viceversa.

In this sense, the objective of the present report was to review data related with in vivo antioxidant activity of phenolic extracts and compounds, highlighting parameters involved in their metabolism, bioavailability and capacity to prevent/avert aging-related diseases.

\section{Human nutrition: nutrients and phytochemicals}

\subsection{Critical perspective}

Dietary practices play an important and unquestionable role at health level. Increasing evidences have shown that certain foods (currently known as functional foods) possess numerous health benefits, being able not only to prevent a wide variety of human disorders, but also to revert and even block degenerative processes (Devasagayam et al., 2004; Espín et al., 2007; Murray \& Pizzorno, 2005). In fact, some of those foods and even dietary practices confer immediate therapeutic actions.

In the last years, numerous changes have been observed in daily diets, in part due to the modern lifestyle and globalization (Goodman et al., 2011; Kaushik, Satya, Khandelwal, \& Naik, 2010). Sensorial gratification is one of the most important points in food choices by individuals, but it does not mean that provides correct nourishment (Murray \& Pizzorno, 2012). Increasing evidences have shown that diets rich in plant foods is protective towards a wide variety of health conditions; and in contrary, diets' providing a low percentage of plant foods are considered a triggering factor to the development of human organisms disorders (Murray \& Pizzorno, 2005, 2012). In fact, a substantial prevalence of chronic and degenerative disorders has been observed, which curiously becomes more frequent with the implementation of globalization and changes in dietary patterns. Metabolic (obesity, gout, diabetes), cardiovascular (high blood pressure, varicose veins, embolisms, strokes, arteriosclerosis, hearth dysfunctions), gastrointestinal (constipation, diverticulitis, bowel inflammatory syndromes, ulcers, haemorrhoids), and auto-immune disorders, and even cancer, are among the most frequent, with the highest rates of incapacity and dependence in worldwide population (Espín et al., 2007; Fernandez-Panchon et al., 2008; Murray \& Pizzorno, 2012; Rubió et al., 2013).

\subsection{From nutritional attributes to effective biological functions}

Considering that enriched-diets in fruits and vegetables have been suggested to provide a dose-dependent protective effect against chronic disorders, numerous studies have been carried out towards to support those evidences (Rubió et al., 2013). Among the numerous publications, researchers have highlighted that fruits and vegetables act as barrier against cancer, branding them as "chemopreventers", due to the presence of bioactive “phytochemicals” (Murray \& Pizzorno, 2012). These plant-derived molecules comprise pigments, such as chlorophyll, carotenes, flavonoids and other phenolic compounds; vitamins; dietary fibers, enzymes, minerals, among other minor constituents (Devasagayam et al., 2004; Grotewold, 2006; Murray \& Pizzorno, 2005). In addition, they establish relations with other plant constituents including those through synergistic mechanisms (Ndhlala, Stafford, \& Staden, 2013). This explains why in many cases whole matrices give a higher protection than isolated/single nutrients. Despite being vestigial components, phytochemicals play a crucial role in preventing and treating illnesses.

Regarding phenolic compounds, increasing evidences prove their protective activity against a multitude of human conditions (Carocho \& Ferreira, 2013; Grotewold, 2006; Mukherjee \& Houghton, 2009). Mainly responsible for colour, smell and even protection of fruits and flowers, these phytochemicals possess really important bioactive attributes. Despite being each class of phenolic compounds mainly responsible for a specific bioactivity, they commonly evidence polyvalence reactions, being even able to strengthen the potential of other compounds, to block side effects of some constituents and also to acquire other biological properties (when combined in whole matrices) (Mukherjee \& Houghton, 2009). Antioxidant activity is one of the most studied bioactive potential of phenolic compounds, in spite of the difficulty to quantify in an accurate mode, mainly through in vitro studies. Numerous variables act as conditioning factors; so, false-positive or negative results could be achieved. For example, phenolic matrices with pronounced in vitro antioxidant activity, could not have the same efficacy when in vivo studies are carried out; but, the opposite effect can also occur (Halliwell, 2012; Heim et al., 2002; Mukherjee \& Houghton, 2009). This fact could be explained mainly due to two main reasons: firstly, in vitro studies do not consider whole human metabolism, biological barriers and the entire chemical reactions of the human organism; on the other hand, some compounds, ingested on their active form lack the bioactive potential after metabolism, while others acquire their bioactive form only after being metabolized (Espín et al., 2007; Heim et al., 2002; Holst \& Williamson, 2008). In addition, it is also important to highlight that biochemical interactions (between phytochemicals and foods/ drugs) could eliminate, reduce and even improve their bioactivity, making these compounds harmful or beneficial to the organisms (Fernandez-Panchon et al., 2008; Holst \& Williamson, 2008).

\subsection{Benefits and constraints beyond human metabolism}

Human organisms need a correct metabolic function in order to ensure a good health and well-being. Through human metabolism, numerous harmful substances (commonly known as free radicals) are produced and, therefore, effective endogenous detoxifying and neutralizing processes need to function properly in order to prevent damages (Devasagayam et al., 2004; Valko et al., 2007). Superoxide dismutase (SOD), catalase (CAT) and glutathione peroxidase (GSH-Px) are the three most important enzymes (considered primary enzymes) involved in the direct elimination of reactive oxygen species- ROS (hydroxyl radical, superoxide radical, hydrogen peroxide, peroxyl radical, hydroperoxide, singlet oxygen), through metabolism of those toxic oxidative intermediates (Devasagayam et al., 2004; Singh et al., 2004; Valko et al., 2007). On the other hand, the secondary enzymes glutathione reductase (GR), glucose-6-phosphate dehydrogenase (G-6-PDH), and glutathioneS-transferase (GST) are involved in the detoxification of ROS by decreasing peroxide levels or maintaining a correct supply of metabolic intermediates (such as glutathione and NADPH), crucial for an optimum functioning of the primary antioxidant enzymes (Singh et al., 2004). Notwithstanding, endogenous metabolism is 
also a source of harmful substances; stress, underlying diseases/ disorders, ingested chemicals and drugs, pesticides and other food contaminants are able to attack organisms. In addition, pollution, modern lifestyle, radiations, inadequate behaviours, dependencies, among other factors comprise important aggressors, which also improve free radicals production (Devasagayam et al., 2004; Singh et al., 2004; Valko et al., 2007).

One of the most important and basic functions of cells is to maintain homeostasis; however, by itself, the proper human metabolism is a triggering factor to cellular unbalance and, consequently, dysfunction. Furthermore, in face of a poor nutritional status and weak ingestion of antioxidant phytochemicals, associated with a continuous exposure to toxic substances (mainly by oral or dermal routes and inhalation), a higher risk to develop disease/dysfunction will be doubtless installed (Valko et al., 2007). In addition, and considering the modern lifestyle, the human endogenous detoxifying and antioxidant processes of the organisms are not able to neutralize the free radicals produced daily. Still more, endogenous antioxidants require an appropriate supplying of micronutrients and other functional biomolecules, which act mainly as cofactors, in order to ensure an optimal functioning (Fardet, Rock, \& Rémésy, 2008; Singh et al., 2004).

In this sense, it is of the utmost importance to provide enrichedfood sources of antioxidants. As referred above, phenolic matrices comprise an extremely rich source of phytochemicals, which present a multitude of health benefits, including their ability to act as free radicals scavengers. Currently, several controversies still exist and no definite consensus has been established on the antioxidant potential of natural matrices (Carocho \& Ferreira, 2013). It is obvious that not all the studied variables can be controlled; itself, this is a sufficient fact, which leads to different conclusions. On the other hand, when in vivo studies are carried out, additional confusing factors are present which cannot be fully controlled. For example, the interference of some foods and even nutrients on the bioavailability and bioactivity of phenolic matrices is doubtless established, with milk being one of the most studied. Several reports have evidenced that milk interferes with the antioxidant potential of enriched-phenolic matrices, including blueberries (Serafini et al., 2009), tea (Langley-Evans, 2000a; Lorenz et al., 2007) and chocolate (Serafini et al., 2003). However, other authors have also reported contradictory results for the same phenolic matrices (Hof, Kivits, Weststrate, \& Tijburg, 1998; Hollman, Hof, Tijburg, \& Katan, 2001; Leenen, Roodenburg, Tijburg, \& Wiseman, 2000). Thus, as in vivo studies are poorly developed, those contradictions remain unclear.

\section{In vitro vs. in vivo studies: challenges and current perspectives}

Medicinal plants have deserved an increasing interest in the last years. In fact, they possess a multitude of health benefits, widely known since pre-historic era. Notwithstanding, due to the absence of scientific validation, they were poorly recommended and even used by worldwide population; but, currently, significant changes have been achieved (Halberstein, 2005). Among the bioactive properties, the antioxidant potential has been one of the most studied, for numerous plant species, different plant parts, extraction procedures and evaluation assays. But even other bioactive properties have been increasingly studied (Dai \& Mumper, 2010; Rubió et al., 2013). Obviously studying the in vitro properties of natural matrices should be the first objective to carry out; but, numerous studies in which promising antioxidant effects were achieved, lack of in vivo validation (Fernandez-Panchon et al., 2008; Rubió et al., 2013). For example, by comparing the number of publications, from 2000 up to 2014, a pronounced increase on the magnitude of studies in which the in vitro and in vivo antioxidant potential of phenolic matrices have been observed. However, still remains existing a significant gap related with the in vivo validation of the antioxidant potential of phenolic extracts. Further, numerous studies report effective in vitro antioxidant potential of natural matrices, in an exponential manner; but in vivo studies are still scarce. Another interesting fact is that, contrarily to in vitro studies, the in vivo experiments do not follow the previously mentioned exponential growth. Normally, in vivo studies are developed by using several animal models; however, to the majority of the tested phenolic matrices, in which significant free radicals scavenging activity was observed, no in vivo studies were carried out to confirm this potential. The study of new phenolic matrices is undoubtedly important, but to deepen its potential, namely through chemical characterization of the bioactive constituents, followed by testing the in vivo potential and related mechanisms of action, if applicable, carrying out clinical trials, is of the utmost importance. It is important to highlight that in vitro methods possess numerous constraints, and for that reason, a significant variation is observed when different methods are used to evaluate the same biochemical parameter. In addition, it is very important not forget that the whole human metabolism gives a significant contribution to the antioxidant potential. Through the entire human metabolism numerous compounds are converted on their active forms, while others are inactivated or even linked to several biomolecules that can change the original effect. None of those aspects could be achieved by using in vitro studies, and apart from that, falsenegative and false-positive results could be achieved. Therefore, in vivo studies should be carried out to confirm the in vitro results.

Besides phenolic matrices, the in vitro and also in vivo antioxidant potential of individual phenolic compounds, derived from natural matrices, has been also carried out. By observing the number of publications, from 2000 up to 2014, in which the in vitro and in vivo antioxidant potential of phenolic compounds was accessed, similarly to phenolic extracts, in vitro studies have increased exponentially, while in vivo experiments remain poorly investigated. Furthermore, despite the evaluation of specific compounds, not least important is to evaluate synergisms, antagonisms, polyvalence reactions and even lack of biological effect in mixtures of those compounds. Individual phenolic constituents exist in different proportions in whole natural matrices; so, the final observed potential is not always the sum of each one of the individual phenolic compounds present.

Currently, numerous phytochemical formulations are available, not only including plant crude extracts but also isolated phenolic compounds, mixtures of them or even with other biological constituents (vitamins, minerals, trace elements, among others). In this sense, apart from the elucidation of the effects of isolated and even mixtures of phenolic compounds derived from natural matrices, through in vitro studies, the development of in vivo experiments is of the major importance. Clinical trials should be also carried out, as well as the validation of numerous phytochemical formulations used for antioxidant purposes.

\section{In vivo antioxidant activity of phenolic extracts/ compounds}

\subsection{Plant phenolic extracts with in vivo antioxidant activity}

Animal models comprise the main focus of the studies towards evaluating in vivo antioxidant activity of phenolic matrices (Table 1). Rats (Wistar and albino Wistar, Sprague Dawley and Wistar-Hannover), followed by mice (Swiss, Kunming and CF1) and Swiss albino inbred mouse, are the most frequently used laboratory animals. Those animals are submitted to a broad spectrum of 
Table 1

Plant species used to obtain phenolic extracts with in vivo antioxidant activity evaluated in animal models.

\begin{tabular}{|c|c|c|c|}
\hline Plant & References & Plant & References \\
\hline Afzelia africana SM & (Atawodi, Iliemene, \& Onyike, 2014) & Ocimum sanctum $\mathrm{L}$. & $\begin{array}{l}\text { (Samson, Sheeladevi, \& Ravindran, } \\
\text { 2007) }\end{array}$ \\
\hline Astragalus sinicus L. & (Lim et al., 2011) & Parkia biglobosa & (Ademiluyi \& Oboh, 2012) \\
\hline Beta vulgaris $\mathrm{L}$. & (Vulić et al., 2014) & Paspalum scrobiculatum L. & (Hegde, Rajasekaran, \& Chandra, 2005) \\
\hline Capsicum baccatum L. var. pendulum & (Kappel et al., 2008) & Passiflora edulis Sims & (Silva et al., 2013) \\
\hline Cassia fistula Linn. & $\begin{array}{l}\text { (Maheep, Sunil, Yogesh, Durgesh, \& Kanika, } \\
\text { 2010) }\end{array}$ & Pinus koraiensis L. & (Su et al., 2009) \\
\hline Ceratonia siliqua & (Hsouna et al., 2011) & Piper guineense Schumach. et Thonn & (Adefegha \& Oboh, 2012) \\
\hline $\begin{array}{l}\text { Choerospondias axillaris (Roxb.) B.L. Burtt } \\
\text { and A.W. Hill }\end{array}$ & (H. Wang, Gao, Zhou, Cai, \& Yao, 2008) & Prosopis juliflora & (Almaraz-Abarca et al., 2007) \\
\hline Clerodendrum infortunatum $\mathrm{L}$. & $\begin{array}{l}\text { (Gouthamchandra, Mahmood, \& Manjunatha, } \\
\text { 2010) }\end{array}$ & $\begin{array}{l}\text { Pseudocydonia sinensis Schneid. var. } \\
\text { Toukarin }\end{array}$ & $\begin{array}{l}\text { (Hamauzu, Inno, Kume, Irie, \& } \\
\text { Hiramatsu, 2006) }\end{array}$ \\
\hline $\begin{array}{l}\text { Coffea arabica }(60 \%) \text { and Coffea canefora var. } \\
\text { robusta }(40 \%) \text { mixture }\end{array}$ & $\begin{array}{l}\text { (Contini, Baccelloni, Frangipane, Merendino, \& } \\
\text { Massantini, 2012) }\end{array}$ & Pueraria lobata (Willd.) Ohwi & (Bebrevska et al., 2010) \\
\hline Cydonia oblonga Mill. cv. Smyrna & (Hamauzu et al., 2006) & Saccharum officinarum $\mathrm{L}$. & $\begin{array}{l}\text { (Duarte-Almeida, Novoa, Linares, } \\
\text { Lajolo, \& Genovese, 2006) }\end{array}$ \\
\hline Dipteryx alata Vog. & (Siqueira et al., 2012) & Saraca indica $\mathrm{L}$. & (Sen et al., 2014) \\
\hline Eleusine coracana (L.) Gaertn. & (Hegde et al., 2005) & Schisandra chinensis & (Cheng et al., 2013) \\
\hline Euryale ferox Salisb. & (Wu et al., 2013) & $\begin{array}{l}\text { Symplocos cochinchnensis var. } \\
\text { laurina }\end{array}$ & (Sunil \& Ignacimuthu, 2011) \\
\hline Ferula szovitsiana DC & $\begin{array}{l}\text { (Dehghan, Shafiee, Ghahremani, Ardestani, \& } \\
\text { Abdollahi, 2007) }\end{array}$ & Torilis leptophylla $\mathrm{L}$. & (Saeed, Khan, \& Shabbir, 2012) \\
\hline Ficus glomerata Roxb. & (Verma, Vijayakumar, Rao, \& Mathela, 2010) & $\begin{array}{l}\text { Vaccinium ashei Reade (Maru and } \\
\text { Centurion varieties) }\end{array}$ & (Molan, Lila, \& Mawson, 2008) \\
\hline Glycine max L. Merrill & (Ademiluyi \& Oboh, 2012) & Vigna subterranea L. Verdc & (Ademiluyi \& Oboh, 2012) \\
\hline Helichrysum plicatum ssp. plicatum & (Aslan, Orhan, Orhan, Sezik, \& Yesilada, 2007) & Vigna unguiculata & (Kapravelou et al., 2015) \\
\hline Hemerocallis fulva Linn. & (Que, Mao, \& Zheng, 2007) & Vitis vinifera varieties & (Gris et al., 2013) \\
\hline Hordeum vulgare $\mathrm{L}$. & (Qingming et al., 2010) & Xylopia aethiopica (Dun.) A. Rich & (Adefegha \& Oboh, 2012) \\
\hline Humulus lupulus L. & (X. Wang, Yang, Yang, \& Tian, 2014) & Zizyphus jujuba cv. Banzao & (H. Zhao, Zhang, \& Yang, 2014) \\
\hline Malus domestica Borkh. cv. Fuji & (Hamauzu et al., 2006) & Zizyphus jujuba cv. Goutouzao & \\
\hline Meyna spinosa Roxb. & (Sen, De, Devanna, \& Chakraborty, 2013) & Zizyphus jujuba cv. Jinsizao & \\
\hline Moringa oleifera Lam. & $\begin{array}{l}\text { (Jaiswal et al., 2013; Verma, Vijayakumar, } \\
\text { Mathela, \& Rao, 2009) }\end{array}$ & Zizyphus jujuba cv. Junzao & \\
\hline Myrciaria dubia McVaugh & $\begin{array}{l}\text { (Gonçalves, Lellis-Santos, Curi, Lajolo, \& } \\
\text { Genovese, 2014) }\end{array}$ & Zizyphus jujuba cv. Pozao & \\
\hline Nelumbo nucifera Gaertn. & $\begin{array}{l}\text { (Huang et al., 2010; Rai, Wahile, Mukherjee, } \\
\text { Saha, \& Mukherjee, 2006) }\end{array}$ & $\begin{array}{l}\text { Zizyphus jujuba cv. Yuzao } \\
\text { Zizyphus jujuba cv. Xiaozao }\end{array}$ & \\
\hline
\end{tabular}

stress-inducing agents, such as ethanol, D-galactose, iron, alloxan, streptozotocin (STZ), carbon tetrachloride $\left(\mathrm{CCl}_{4}\right)$, bromobenzene, radiation, noise exposure, methylmercuric chloride $(\mathrm{MeHgCl})$, tertbutyl hydroperoxide $(t-B O O H)$ and hydrogen peroxide $\left(\mathrm{H}_{2} \mathrm{O}_{2}\right)$.

Acetone, chloroform, ethanol, ethyl acetate, methanol, water, ethanol:water, acetone:water: $\mathrm{HCl}$, petroleum ether, and methanol: $1 \mathrm{~N} \mathrm{HCl}$ were used as solvents to obtain the different phenolic extracts tested. Plant species belonging to Leguminoseae and Rhamnaceae, followed by Fabaceae, Poaceae, Rubiaceae, Nymphaeaceae, Rosaceae and Moringaceae have been mostly studied as sources of phenolic extracts. However, Anacardiaceae, Annoneaeceae, Apiaceae, Asteraceae, Cannabaceae, Chenopodiaceae, Ericaceae, Lamiaceae, Liliaceae, Moraceae, Myrtaceae, Passifloraceae, Pinaceae, Schisandraceae, Solanaceae, Symplococaceae, Umbeliferae, Verbenaceae and Vitaceae have also been used.

The evaluation of the in vivo antioxidant potential is mainly assessed by the effects on different biochemical parameters directly involved in the maintenance of a balanced/correct antioxidant status, such as: malondialdehyde (MDA), CAT, SOD, glutathione (GSH), GSH-Px, GST, GR, xanthine oxidase (XOD), peroxidase (Px), alanine aminotransferase (ALT), aspartate aminotransferase (AST), lactate dehydrogenase (LDH), alkaline phosphatase (ALP), c-Glutamyl transpeptidase (c-GT), ferric reducing antioxidant power (FRAP), 2,2'-azobis(2-amidino-propane) dihydrochloride (APPH)hemolysis, protein carbonylation (PC), diene conjugates, collagen glycation, total antioxidant capacity (TAOC), monoamine oxidase (MAO), oxidative hemolysis, oxygen radical absorbance capacity (ORAC), gamma glutamyl transferase (GGT), glutamate oxaloacetate transaminases (GOT), glutamate pyruvate transaminases (GPT) and 2,2-azino-bis(3-ethyl-benzothiazoline-6-sulphonate) (ABTS).

Table 2 shows the in vivo antioxidant potential of phenolic extracts, evaluated through clinical trials, using healthy human volunteers or groups of individuals in special physiological conditions, namely basketball players during training (Chang et al., 2007), postmenopausal women (Lorenz et al., 2007) and human prediabetic Mauritians (Somanah, Bourdon, Rondeau, Bahorun, \& Aruoma, 2014). Among of the studied plant families only five of them were also previously studied in animal models: Asteraceae, Ericaceae, Myrtaceae, Rosaceae and Vitaceae, but not the same extract types.

Lactuca sativa L. and Helichrysum plicatum ssp. plicatum capitulums were the studied Asteraceae plant extracts tested in human volunteers and Wistar-albino rats, respectively. Among to the Ericaceae family, Vaccinium angustifolium Ait. and Vaccinium corymbosum L. were studied in healthy human volunteers, while Vaccinium ashei (Mau and Centurion varieties) was tested in Sprague Dawley rats. In the Myrtaceae family, Ugni molinae Turcz. and Myrciaria dubia McVaugh were studied in human healthy volunteers and STZ-induced diabetic Wistar rats, respectively. In relation to Rosaceae family, while Fragaria ananassa Duch was tested in human healthy volunteers, Cydonia oblonga Mill. cv. Smyrna, Malus domestica Borkh. cv. Fugi and Pseudocydonia sinenses Schneid. var. Toukarin were evaluated in healthy Wistar rats. Lastly, among the Vitaceae family, Vitis vinifera L. extract was tested in human healthy volunteers, and different $V$. vinifera varieties were also studied in healthy Swiss mice. Other plant families tested in human volunteers include Caricaceae, Convolvulaceae, Malvaceae and Theaceae. Total radical trapping parameter (TRAP), FRAP, total antioxidant 
Table 2

Plant phenolic extracts with in vivo antioxidant activity evaluated in clinical trials with human healthy volunteers.

\begin{tabular}{|c|c|c|c|c|c|c|c|}
\hline Family & Plant & Preparation & Concentration & Dosage & Period & Assays & References \\
\hline Asteraceae & $\begin{array}{l}\text { Lactuca sativa } \\
\text { L. }\end{array}$ & $\begin{array}{l}\text { Fresh lettuce } \\
\text { Stored lettuce }\end{array}$ & 250 g/day & Single dose & 1 day & TRAP & (Serafini et al., 2002) \\
\hline Caricaceae & $\begin{array}{l}\text { Carica papaya } \\
\text { L. }\end{array}$ & Fermented ripe pulp & $30 \mathrm{mg} / \mathrm{mL}$ & 1 dose $(200 \mathrm{~mL}) /$ day & 14 weeks $^{\mathrm{a})}$ & $\begin{array}{l}\text { TAS, inhibition of } \\
\text { hemolysis and level of PC }\end{array}$ & $\begin{array}{l}\text { (Somanah et al., } \\
\text { 2014) }\end{array}$ \\
\hline \multirow[t]{2}{*}{ Convolvulaceae } & $\begin{array}{l}\text { Ipomoea } \\
\text { batatas (L.) }\end{array}$ & Cooked leaves & 200 g/day & 2 doses (each $100 \mathrm{~g}$ ) & 7 weeks & $\begin{array}{l}\text { TAS, GSH, 8-OHdG, MDA, 4- } \\
\text { HNE }\end{array}$ & (Chen et al., 2008) \\
\hline & Lam. & & & Single dose & $\begin{array}{l}2 \text { periods (each } \\
\text { lasting } 2 \\
\text { weeks) }^{\mathrm{b})}\end{array}$ & $\begin{array}{l}\text { TAS, lipid hydroperoxides, } \\
\text { 8-OHdG, MDA }\end{array}$ & (Chang et al., 2007) \\
\hline \multirow[t]{2}{*}{ Ericaceae } & $\begin{array}{l}\text { Vaccinium } \\
\text { angustifolium } \\
\text { Ait. }\end{array}$ & $\begin{array}{l}\text { Freeze-dried wild } \\
\text { strawberry dispersed } \\
\text { in water }\end{array}$ & $200 \mathrm{mg} / \mathrm{mL}$ & 1 dose/day & 7 days & ORAC and TAS & (Kay \& Holub, 2002) \\
\hline & $\begin{array}{l}\text { Vaccinium } \\
\text { corymbosum } \\
\text { L. }\end{array}$ & Fresh fruits & 200 g/day & 1 dose/week & 2 weeks & TAS, FRAP and TRAP & (Serafini et al., 2009) \\
\hline \multirow[t]{2}{*}{ Malvaceae } & $\begin{array}{l}\text { Theobroma } \\
\text { cacao L. }\end{array}$ & $\begin{array}{l}\text { M\&M's Semi- Sweet } \\
\text { Chocolate Mini } \\
\text { Baking Bits }\end{array}$ & $\begin{array}{l}105 \mathrm{~g} \text { ( } 80 \mathrm{~g} \text { of } \\
\text { procyanidin-rich } \\
\text { chocolate) }\end{array}$ & Single dose & 1 day & TBARS, TRAP and LCL & (D. Rein et al., 2000) \\
\hline & & Commercial product & $100 \mathrm{~g}$ & Single dose & 1 day & FRAP & (Serafini et al., 2003) \\
\hline Myrtaceae & $\begin{array}{l}\text { Ugni molinae } \\
\text { Turcz. }\end{array}$ & Infusion of leaves & $10 \mathrm{mg} / \mathrm{mL}$ & 2 doses $(100 \mathrm{~mL}) /$ day & 3 days & TBARS, and TEAC-CUPRAC & (Avello et al., 2013) \\
\hline Oleaceae & Olea europaea & Fresh fruits & 100 g/day & Single dose & $24 \mathrm{~h}$ & $\begin{array}{l}\text { TAS, plasma total } \\
\text { polyphenol assay }\end{array}$ & $\begin{array}{l}\text { (Kountouri et al., } \\
\text { 2007) }\end{array}$ \\
\hline Rosaceae & $\begin{array}{l}\text { Fragaria } x \\
\text { ananassa, } \\
\text { Duch. }\end{array}$ & Fresh fruit & $500 \mathrm{~g}$ & 2 doses $(250 \mathrm{~g}) /$ day & 16 days & $\begin{array}{l}\text { FRAP and TEAC, } \\
\text { erythrocytes hemolysis }\end{array}$ & (Tulipani et al., 2011) \\
\hline \multirow[t]{9}{*}{ Theaceae } & $\begin{array}{l}\text { Camelia } \\
\text { sinensis (L.) }\end{array}$ & Black tea & $16.25 \mathrm{mg} / \mathrm{mL}$ & Six doses $(200 \mathrm{~mL})$ & 1 day & FRAP & $\begin{array}{l}\text { (Langley-Evans, } \\
\text { 2000b) }\end{array}$ \\
\hline & Kuntze & & $6.67 \mathrm{mg} / \mathrm{mL}$ & Single dose $(300 \mathrm{~mL})$ & 1 day & FRAP & (Leenen et al., 2000) \\
\hline & & & $20 \mathrm{mg} / \mathrm{mL}$ & Single dose $(300 \mathrm{~mL})$ & 1 day & TRAP & $\begin{array}{l}\text { (Serafini, Laranjinha, } \\
\text { Almeida, \& Maiani, } \\
\text { 2000) }\end{array}$ \\
\hline & & & $3.33 \mathrm{mg} / \mathrm{mL}$ & $\begin{array}{l}\text { Eight doses }(150 \mathrm{~mL}) / \text { day, } \\
\text { every } 2 \mathrm{~h}\end{array}$ & 3 days & $\begin{array}{l}\text { Plasma concentration } \\
\text {-time curve of quercetin or } \\
\text { kaempferol }\end{array}$ & $\begin{array}{l}\text { (Hollman et al., } \\
\text { 2001) }\end{array}$ \\
\hline & & & $10 \mathrm{mg} / \mathrm{mL}$ & Single dose $(500 \mathrm{~mL})$ & 1 day $^{\mathrm{c})}$ & $\begin{array}{l}\text { Endothelial function (FMD } \\
\text { and NMD) }\end{array}$ & (Lorenz et al., 2007) \\
\hline & & Green tea & $6.67 \mathrm{mg} / \mathrm{mL}$ & Single dose (300 mL) & 1 day & FRAP & (Leenen et al., 2000) \\
\hline & & & $20 \mathrm{mg} / \mathrm{mL}$ & Single dose $(300 \mathrm{~mL})$ & 1 day & TRAP & (Serafini et al., 2000) \\
\hline & & & $16.67 \mathrm{mg} / \mathrm{mL}$ & $\begin{array}{l}1^{\text {st }} \text { week }(150 \mathrm{~mL}), 2 \text { nd week } \\
(300 \mathrm{~mL}), 3 \mathrm{rd} \text { week } \\
(450 \mathrm{~mL}) / \text { day }\end{array}$ & 3 weeks & TAS & (Sung et al., 2000) \\
\hline & & & $3.33 \mathrm{mg} / \mathrm{mL}$ & $\begin{array}{l}\text { Eight doses }(150 \mathrm{~mL}) / \text { day, } \\
\text { every } 2 \mathrm{~h}\end{array}$ & 3 days & $\begin{array}{l}\text { Plasma concentration } \\
\text {-time curve of quercetin or } \\
\text { kaempferol }\end{array}$ & $\begin{array}{l}\text { (Hollman et al., } \\
\text { 2001) }\end{array}$ \\
\hline \multirow[t]{4}{*}{ Vitaceae } & $\begin{array}{l}\text { Vitis vinifera } \\
\text { L. }\end{array}$ & $\begin{array}{l}\text { White wine } \\
\text { dealcoholized }\end{array}$ & $20 \mathrm{mg} / \mathrm{mL}$ & Single dose $(300 \mathrm{~mL})$ & 1 day & TRAP & (Serafini et al., 2000) \\
\hline & & $\begin{array}{l}\text { Red wine } \\
\text { dealcoholized }\end{array}$ & $20 \mathrm{mg} / \mathrm{mL}$ & Single dose $(300 \mathrm{~mL})$ & 1 day & TRAP & (Serafini et al., 2000) \\
\hline & & Whole grapes & $250 \mathrm{~g}$ & 1 dose/day & 4 weeks & ORAC-PCA & (Parker et al., 2007) \\
\hline & & Whole raisins & $\begin{array}{l}50 \mathrm{~g} \text { of golden } \\
\text { raisins } \\
50 \mathrm{~g} \text { sun dried- } \\
\text { raisins }\end{array}$ & 1 dose/day & 4 weeks & & (Parker et al., 2007) \\
\hline
\end{tabular}

\footnotetext{
${ }^{a}$ Human pre-diabetic Mauritians.

b Basketball players during training.

c Postmenopausal women; FMD, flow-mediated dilatation; NMD, nitro-mediated dilatation.
}

status (TAS), ORAC, luminal-enhanced chemiluminescence measurement (LCL), PC, GSH, MDA, 8-hydroxy-2-deoxyguanosine (8OHdG), 4-hydroxynonenal (4-HNE), trolox equivalent antioxidant capacity-cupric ion reducing antioxidant capacity (TEAC-CUPRAC), endothelial function, lipid hydroperoxides and inhibition of hemolysis were the most commonly methods to evaluate human biochemical parameters (as shown in the Table 2).

As previously mentioned, for the majority of the plant extracts tested in animal models, in which pronounced antioxidant properties were observed, no clinical trials involving human volunteers were carried out. Nevertheless, it is important to highlight that the achievement of a significant antioxidant potential in animal models (rats, mice, mouse, etc.) does not mean that the same potential will be found in human individuals. It is important to clearly understand the real effects of plant phenolic extracts, their bioactive individual compounds and mechanisms of action, and even to establish prophylactic and therapeutic doses, not only in animal models but also in human individuals.

\subsection{Phenolic compounds with in vivo antioxidant activity}

Table 3 shows the in vivo antioxidant potential of individual phenolic compounds, by using animal models. Anthocyanins, followed by hydroxybenzoic and hydroxycinnamic acids comprise the 
Table 3

Phenolic compounds with in vivo antioxidant activity evaluated in animal models.

\begin{tabular}{|c|c|c|c|}
\hline Tested compound & Origin & Time of exposure & References \\
\hline $\begin{array}{l}\text { Flavan-3 -ols } \\
(-) \text {-Epicatechin } \\
\text { Flavones }\end{array}$ & Commercial & $90 \mathrm{~min}$ & (Hamauzu et al., 2006) \\
\hline $\begin{array}{l}\text { Apiin } \\
\text { Flavonols }\end{array}$ & Apium graveolens L. var. dulce (leaves) & 28 days & (P. Li et al., 2013) \\
\hline Rutin & Commercial & $\begin{array}{l}2 \mathrm{~h} \\
28 \text { days } \\
2 \mathrm{~h}\end{array}$ & $\begin{array}{l}\text { (Sen et al., 2013) } \\
\text { (P. Li et al., 2013) } \\
\text { (Sen et al., 2014) }\end{array}$ \\
\hline Hydroxycinnamic acids & & & \\
\hline 5-caffeoylquinic acid & Commercial & $90 \mathrm{~min}$ & (Hamauzu et al., 2006) \\
\hline Ferulic acid & $\begin{array}{l}\text { Corn bran } \\
\text { Commercial }\end{array}$ & $\begin{array}{l}10 \text { days } \\
14 \text { days } \\
21 \text { days }\end{array}$ & $\begin{array}{l}\text { (Z. Zhao et al., 2005) } \\
\text { (Yeh \& Yen, 2006) } \\
\text { (Fetoni et al., 2010) }\end{array}$ \\
\hline$p$-coumaric acid & Corn bran & $\begin{array}{l}14 \text { days } \\
10 \text { days }\end{array}$ & $\begin{array}{l}\text { (Yeh \& Yen, 2006) } \\
\text { (Z. Zhao et al., 2005) }\end{array}$ \\
\hline Hydroxybenzoic acids & & & \\
\hline $\begin{array}{l}\text { Gallic acid } \\
\text { Gentisic acid } \\
\text { p-hydroxy-benzoic acid }\end{array}$ & Commercial & $\begin{array}{l}14 \text { days } \\
14 \text { days } \\
14 \text { days }\end{array}$ & (Yeh \& Yen, 2006) \\
\hline $\begin{array}{l}\text { Protocatechuic acid } \\
\text { Coumestans }\end{array}$ & Alpinia oxyphylla Miq. & 9 days & (Shi et al., 2006) \\
\hline $\begin{array}{l}\text { Pyrocatechol } \\
\text { Anthocyanins }\end{array}$ & Commercial & 1 day & (Roche \& Bogé, 2000) \\
\hline $\begin{array}{l}\text { Cyanidin 3-glucoside } \\
\text { Delphinidin 3-glucoside } \\
\text { Malvidin 3-glucoside } \\
\text { Peonidin 3-glucoside } \\
\text { Petunidin 3-glucoside }\end{array}$ & Abies koreana E.H.Wilson & 2 weeks & (Ramirez-Tortosa et al., 2001) \\
\hline $\begin{array}{l}\text { Anthocyanin } \\
\text { Diferuloylmethanes }\end{array}$ & Ipomoea batatas (L.) Lam. & 30 days & (J. G. Zhao, Yan, Lu, \& Zhang, 2013) \\
\hline Curcumin & Commercial & $\begin{array}{l}6 \text { weeks } \\
31 \text { days }\end{array}$ & $\begin{array}{l}\text { (Gao et al., 2013) } \\
\text { (Xie et al., 2014) }\end{array}$ \\
\hline $\begin{array}{l}\text { Simple phenols } \\
\text { Hydroquinone } \\
\text { Phenol } \\
\text { Resorcinol }\end{array}$ & Commercial & 1 day & (Roche \& Bogé, 2000) \\
\hline
\end{tabular}

most studied phenolic classes. Flavones, flavonols, coumestans, diferuloylmethanes and simple phenols, were also studied. Similarly to the phenolic extracts, rats (Wistar, Sprague-Dawley, Rowett Hooded Lister strain) and mice (ICR, Kunming) are the most commonly used animal models, followed by Hartley albino guineapigs and the marine fish Dicentrarchus labrax. The measured biochemical parameters include MDA, CAT, SOD, GSH, GSH-Px, Px, ALT, AST, AAPH, TAOC, ORAC, 4-HNE, heme oxygenase-1 (HO-1), lipid hydroperoxides, 8-OHdG, learning and memory abilities, auditory brain response, immunohistochemical and western blot analysis (Nrf2, NQO1 and HO-1), as well as the recovery levels of ferulic (FA) and p-coumaric (PCA) acids, and related metabolites.

Since 2010, a significant improvement of the in vitro studies was observed, in which the antioxidant potential of phenolic compounds has been studied; however, a small portion evaluated its in vivo potential. The most tested phenolic compounds are purchased from commercial sources, while those isolated from natural matrices are considerably scarce. Leaves of Apium graveolens L. var. dulce (apiin), corn bran (FA and PCA), Alpinia oxyphylla Miq. (protocatechuic acid), Abies koreana E.H. Wilson (cyanidin 3-glucoside, delphinidin 3-glucoside, malvidin 3-glucoside, peonidin 3glucoside and petunidin 3-glucoside) and Ipomoea batatas (L.) Lam. (anthocyanin) seems to be the main phenolic matrices from which phenolic compounds were isolated, towards the evaluation of in vivo antioxidant effects. It is a fact that numerous phenolic compounds are common in several plant species, but the existence of tenuous differences on their relative abundance is, normally, predictor of the bioactive potential (Avello et al., 2013; Naghdi Badi, Yazdani, Ali, \& Nazari, 2004; Parker, Wang, Pazmiño, \& Engeseth,
2007; Wojdylo, Oszmianski, \& Czemerys, 2007).

Numerous and significant advances have been reported, highlighting the antioxidant potential of individual phenolic compounds. Among them, apigenin, quercetin, kaempferol, myricetin, luteolin, isorhamnetin, neochlorogenic, caffeic, ferulic and $p$-coumaric acids, and their derivatives are the most reported (Heim et al., 2002; Sulaiman, Sajak, Ooi, \& Seow, 2011; Wojdylo et al., 2007). So, and despite the evidences of promising antioxidant potential, mainly through in vitro experiments, it is urgent to validate the in vivo activity of these and other phenolic constituents of plant species. In addition, apart from the effect of slight differences on the phenolic composition, geographical and environmental variations, harvesting time, storage and even extraction procedures are also able to produce considerable variations in phenolic plant contents, and consequently on their total antioxidant potential. Several studies have also reported that different culture conditions are able to produce significant changes on the phenolic composition of the same plant (Avello et al., 2013; Farhat, Landoulsi, Chaouch-Hamada, Sotomayor, \& Jordán, 2013; Sulaiman et al., 2011).

By analysing Fig. 1 reporting studies in which phenolic compounds were evaluated for in vivo antioxidant potential in human volunteers, other significant constraints could be observed. Firstly, from the in vivo experiments in animal models, only three of the studied phenolic compounds were further studied in human volunteers: gallic acid, ferulic acid and rutin. The relative antioxidant efficiency (RAE) of those compounds varied between 17.2 and $27.0 \%$, while for the others, RAE was lower than that. Only ellagic acid $(\mathrm{RAE}=109.9 \%)$, caffeic acid $(\mathrm{RAE}=40.2 \%)$, epigallocatechin gallate $(\mathrm{RAE}=43.1 \%)$, eriodictyol $(\mathrm{RAE}=40.8 \%)$, taxifolin $(30.7 \%)$, 


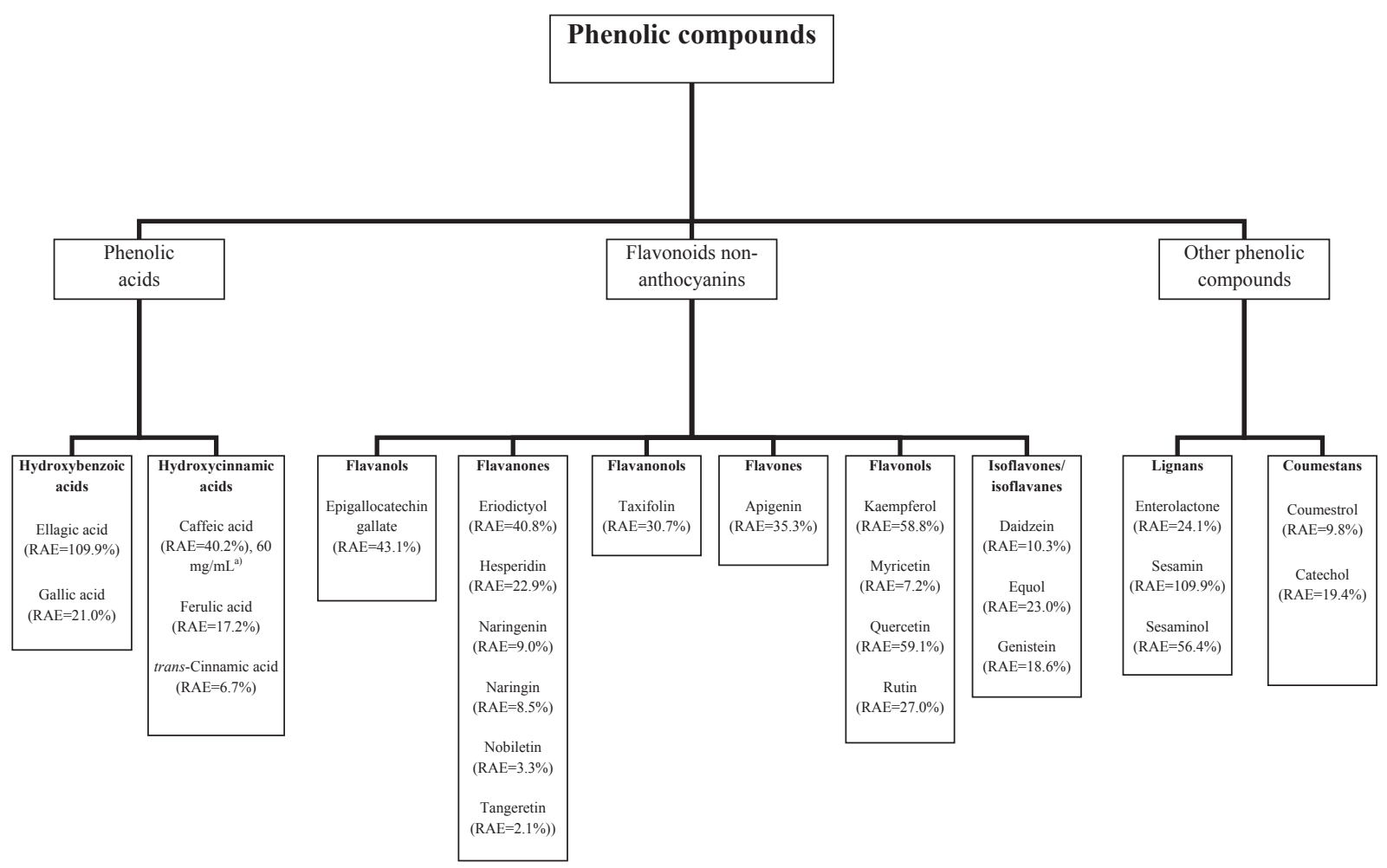

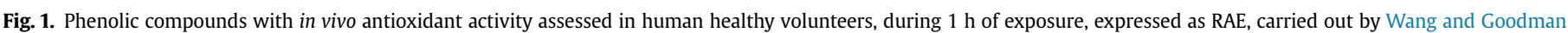

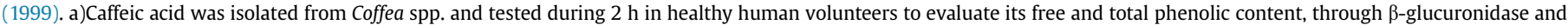
alkaline hydrolysis procedures.

apigenin ( $\mathrm{RAE}=35.3 \%)$, kaempferol ( $\mathrm{RAE}=58.8 \%$ ), quercetin $(\mathrm{RAE}=59.1 \%)$, sesamin $(\mathrm{RAE}=109.9 \%)$ and sesaminol $(\mathrm{RAE}=56.4 \%)$ evidenced a higher potential. However, as previously mentioned, it is important to highlight that those compounds are widely recognized for their antioxidant properties, being even used as food preservatives. Additionally, some researchers also use them as positive controls, in order to assess the bioactive properties of other compounds/substances. Interestingly, Wang and Goodman (1999) only used commercial compounds on their experiment, remaining unclear the real antioxidant effect of these phenolic compounds, isolated from natural sources, as well as their bioavailability and bio-efficacy. In fact, several reports have shown that the recovery of phenolic compounds in plasma, urine and faeces, after ingestion of enriched-natural matrices in phenolic compounds, is poor (Serafini et al., 2002; Z. Zhao et al., 2009; Z. Zhao, Egashira, \& Sanada, 2005).

In relation to the in vivo antioxidant potential of anthocyanins from Vaccinium angustifolium Ait., tested in human volunteers (Fig. 2), only four of them were previously studied in animal models, but isolated from a different natural source (A. koreana E.H. Wilson). Cyanidin 3-O-glucoside, delphinidin 3-O-glucoside, malvidin 3-O-glucoside and petunidin 3-O-glucoside were the isolated anthocyanins from natural sources in which in vivo experiments, both using animal models and human healthy volunteers, were carried out. Curiously, A. koreana E.H. Wilson presented a higher relative abundance of anthocyanins than Vaccinium angustifolium Ait.: $1.3625 \mathrm{~g}$ total antocyanins/100 g (Ramirez-Tortosa et al., 2001) and $1.20 \mathrm{~g}$ total anthocyanins/100 g (Mazza, Kay, Cottrell, \& Holub, 2002), respectively. Considering these results, it is important to highlight that numerous matrices with in vitro and in vivo significant antioxidant potential, could be highly effective and bioavailable, in human individuals. But, as no clinical trials are available for the majority of them, their effect remains questionable.

\section{Bioavailability and bio-efficacy: conditioning factors}

Plant phytochemicals possess numerous health benefits, and despite the chemical composition of each species determines/predicts the final bioactive potential, geographical, environmental and culture conditions, harvesting time, storage, extraction procedures, type of solvents and plant parts used, also have a pronounced impact (Naghdi Badi et al., 2004; Taârit et al., 2012). In fact, several studies have described the effect of each one of the previously described triggering factors, being in vitro studies the most frequently used for this purpose. Besides, it has also been described that several phenolic matrices with in vitro bioactive potential, particularly anti-oxidative effects, do not present the same potential throughout in vivo studies, and vice-versa. Furthermore, the presence of these phenolic compounds in free or bound forms directly influences their potential. For example, Germano et al. (2006) evaluated the in vivo antioxidant potential of phenolic acids, isolated from Trichilia emetica Vahl., in male Wistar rats, and observed significant differences on the phenolic contents, directly dependent from the type of applied treatment (alkaline hydrolysis vs. no treatment). Then, using the free and bound phenolic extract forms, pronounced variations on their ability to inhibit methyl linoleate (MeLo) autoxidation were observed: phenolic extracts submitted to alkaline hydrolysis presented a higher antioxidant potential. Similar results were achieved in the ascorbate/ $\mathrm{Fe}^{2+}-$ induced lipid peroxidation assay. Afterwards, the phenolic acids content was measured in plasma, before and after $\beta$-glucoronidase treatment; the authors observed that the most abundant phenolic acids (caffeic, $p$-coumaric, protocathecuic, vanillic and galic acids), appeared in higher concentration in plasma in comparison with 


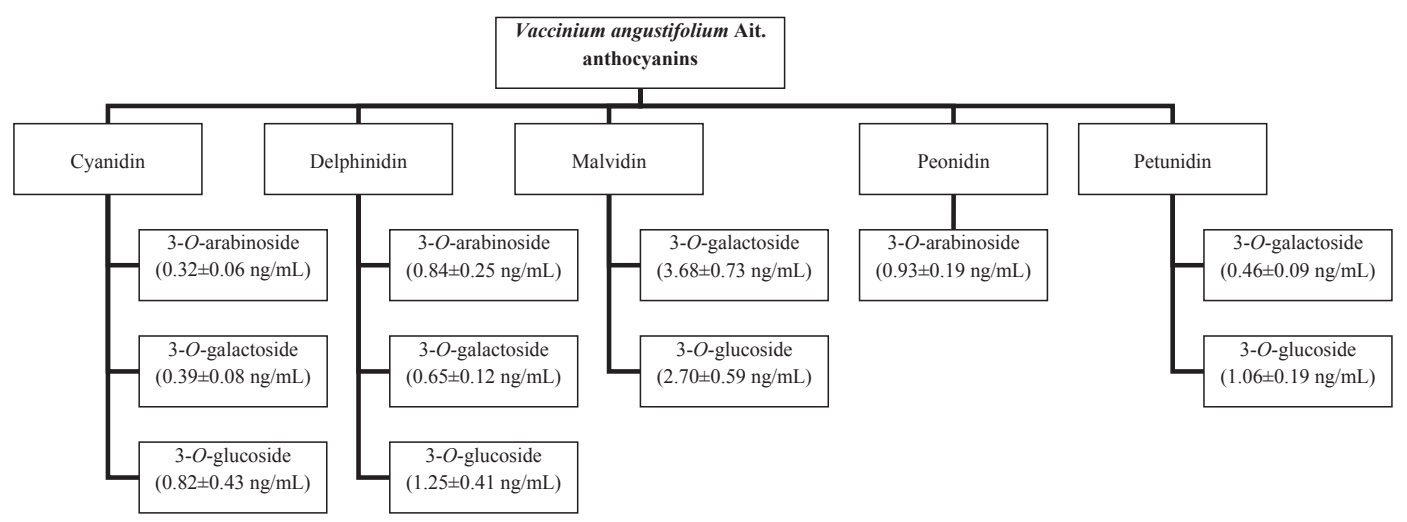

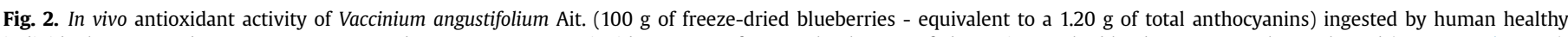

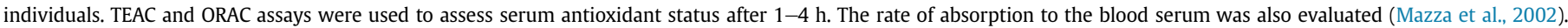
Results are expressed as mean values of the serum anthocyanins concentrations, $4 \mathrm{~h}$ after ingestion of $V$. angustifolium.

hydrolyzed phenolic extracts (Germanò et al., 2006). Taking into account the obtained results, it is evident that some phenolic extracts and, therefore, phenolic compounds, need to be metabolized to be biologically active. Currently, there are no doubts about the importance of the assessment of the in vivo biological potential in phenolic matrices with significant in vitro potential. Fardet et al. (2008), reported similar results, namely the effects of metabolism on the bioavailability of whole-grain cereals and cereal products. In contrast, other authors have reported that some phenolic extracts and even phenolic compounds display a lower bioavailability after ingestion (Z. Zhao et al., 2005).

There are increasing evidences that important food-phenolic compounds interactions affect their bioavailability, and consequently biological potential. One of the most studied are milk-phenolic interactions (Fernandez-Panchon et al., 2008). For example, Serafini et al. (2009) observed that the ingestion of Vaccinium corymbosum $\mathrm{L}$. (blueberries) fruit results in a significant increase of the endogenous plasma antioxidant defences, and also caffeic and ferulic acids level. In addition, they observed that, postingestion, caffeic acid was found in plasma, but not in the food matrix. It means that metabolism favours its absorption and appearance in the circulatory system. Otherwise, the ingestion of blueberries in association with milk impaired the in vivo antioxidant properties of blueberries, and reduced the absorption of caffeic acid (Serafini et al., 2009). But, numerous controversies still remain unclear; while some authors described that milk impairs the bioavailability of phenolic extracts/compounds (Langley-Evans, 2000b; Serafini et al., 2003, 2009), other concluded that no significant interactions were observed (Hof et al., 1998; Hollman, VanHet, Tijburg, \& Katan, 2001; Leenen et al., 2000).

Bioavailability is commonly defined as the amount of a food constituent that is present in the gut, as a consequence of the release of this constituent from the solid food matrix, and may be able to pass through the intestinal barrier (Saura-Calixto, Serrano, \& Goñi, 2007). Different steps directly contribute to this process, namely liberation, absorption, distribution, metabolism and elimination. As previously cited, and despite several variables each one determining the previous steps, inter-individual variations possess a large influence on these processes (M. J. Rein et al., 2013). One of the first and most important factors in the bioavailability is the bioaccessibility: fraction of a compound, which is released from the food matrix in the gastrointestinal lumen and that becomes available for intestinal absorption (M. J. Rein et al., 2013), or the amount of compound reaching the enterocyte in a form suitable for absorption (Scholz \& Williamson, 2007). From a nutritional perspective, the composition of the digested food matrix, the existence of synergic and antagonistic reactions between the different components, and also physicochemical properties $(\mathrm{pH}$, temperature, texture, etc.) influence this process, apart from the mastication (M. J. Rein et al., 2013). Particularly to the plant foods, another special consideration needs to be highlighted. The composition of plant cell wall is markedly resistant to degradation in the upper gut that, therefore, makes difficult the liberation of the active constituents, such as phenolic compounds. Those facts were previously discussed and widely confirmed by some authors. In fact, different reports have stated the benefits of hydrolysis, fermentation, dissolution and even mixture of different phenolic matrices in the improvement of polyphenols bioavailability and its biological potential (Dey \& Kuhad, 2014; Fardet et al., 2008; Kountouri, Mylona, Kaliora, \& Andrikopoulos, 2007; Pinelo, Landbo, Vikbjerg, \& Meyer, 2006; Scalbert et al., 2000). But, extensive studies, which aim to understand the real interference of those methodologies on the improvement of phenolic matrices bioavailability, are significantly scarce. However, it is important to refer that this is a key step to ensure the bio-efficacy of plant bioactive compounds (M. J. Rein et al., 2013). So, it is crucial to enhance the knowledge in this area.

Until now, apart from the bioacessibility, the absorption of these compounds can be also influenced by solubility, interaction with some dietary constituents, molecular changes, protein transporters, human organisms metabolism and, lastly, effects of gut microbiota (M. J. Rein et al., 2013). Those factors, considered the most important determinants to the bioavailability, and then bio-efficacy of bioactive compounds, are schematically described in Fig. 3. Plant bioactive compounds need to be bioavailable to exert beneficial effects in order to provide a significant improvement of health and well-being of the worldwide population, mainly by reducing the frequency of oxidative-stress related diseases/disorders.

\section{Aging-related diseases: evidences of the in vivo role of phenolic antioxidants}

The improvement of the life expectancy and longevity comprises one of the most important purposes of human individuals. Over decades, aging was considered a natural process, i.e. a normal phase, in which a progressive decrease in function, degeneration and malfunction of cells is observed; therefore, cells start to die at a faster rate than new ones are generated. In fact, birth, growth and death markedly represent the three most significant phases of the organisms' life cycle (Murray \& Pizzorno, 2012). However, with the advances of science and technology, new achievements have been stated. 


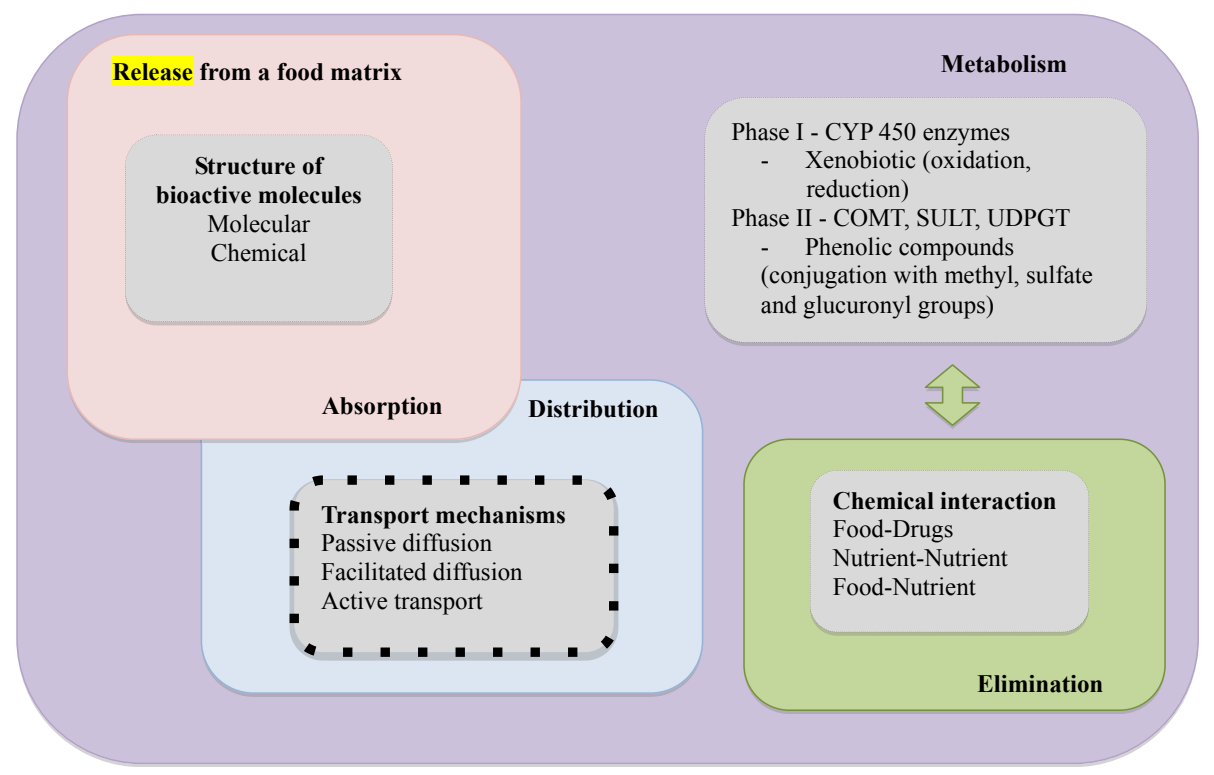

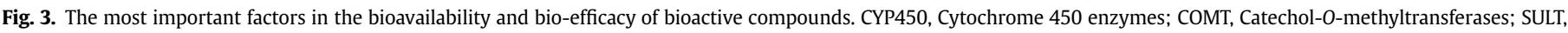
Sulphotransferases; UDPGT, Uridine-5'-diphosphate glucuronosyl-transferases.

The modern definition of aging comprises a progressive postmaturation decline in physiological capacity, accompanied by an increased susceptibility to disease and mortality risk (Ergin, Hariry, \& Karasu, 2013). In fact, the common aging process is associated with a progressive cell failure and, consequently, improves the probability of occurrence of dysfunction/disease/disorders. Notwithstanding, numerous causes of premature aging and cell death have been pointed out during the past decades. It is convenient to highlight that formerly the poor health and hygiene conditions, and medical assistance impaired significantly the life expectancy and increased infant mortality. Currently, in parallel with the improvement of life expectancy, increasing rates of premature aging and death have also been observed (Chaturvedi \& Beal, 2013; Murray \& Pizzorno, 2012). However, cardiovascular, neurodegenerative, gastrointestinal and metabolic diseases, and even cancer comprise the main causes of morbidity and mortality (Valko et al., 2007). More recently, auto-immune, respiratory and osteoarticular disorders have increased their frequency (Musumeci, Szychlinska, \& Mobasheri, 2015; Rubió et al., 2013; Valko et al., 2007).

Pollution, smoking, obesity, sedentary lifestyle, poor nutrition and nutritional deficiencies are pointed as the main contributor factors. It appears a paradox, because in association with greater dietary availabilities and food choices, higher rates of nutritional deficiencies and human disorders/diseases are observed (Murray \& Pizzorno, 2012). Per se, the physical exercise is a triggering factor that accelerates the aging process, while rich source of free radicals; but physical inactivity too, besides to be more dangerous. Apart from the importance of stress management, physical activity, health-promoting lifestyle, and dietary choices are of utmost importance.

All of the most important causes of death are directly related with dietary practices. Dairy products, meat and cereals comprise the base of nutrition to the majority of individuals, while fruits and vegetables/plant products are consumed in a lower frequency than expected. Extremely rich in vitamins, minerals, trace elements, fibers, and also numerous phytochemicals, among them phenolic compounds, those natural matrices have proved to be promising prophylactic and therapeutic agents, widely known in traditional medicine since ancient times (Vanaclocha \& Cañigueral, 2003). Particularly in relation to their antioxidant activity, those plant phytochemicals act not only as free radical scavengers, metal chelators, hydrogen donators and oxygen quenchers, but also they recycle other antioxidant compounds, promote an adequate homeostasis and proper enzymatic function, among other benefits (some of them are not yet discovered) (Carocho \& Ferreira, 2013; Dai \& Mumper, 2010; Li et al., 2014).

Apart from the extensive knowledge about the antioxidant activity of natural matrices, mainly obtained from in vitro studies, recently, new advances regarding in vivo studies have been shared. A significant part of them, have focused on the study of phenolic matrices and not so much on isolated/individual compounds. From the in vivo studies, a great part of phenolic matrices exerts its effects by inhibition of the ROS formation and reduction of oxidative stress, as also by preventing tissue damages (mainly when oxidative stress-inducers are administered) (Shi, An, Jiang, Guan, \& Bao, 2006; Silva et al., 2013; Su, Wang, \& Liu, 2009). The effects on gut microbiota are also being increasingly studied; in fact, not only several phenolic matrices are converted on their active forms but also they exert directional modulatory activities on the gut microbiota, by inhibition of opportunistic flora without interfering with the beneficial bacteria. However, the mechanisms of action are not completely understood (Cueva et al., 2010; Silva et al., 2013; Velderrain-Rodríguez et al., 2014). On the other hand, several isolated phytochemicals, for example, curcumin, exert their effects by causing arsenic methylation and improvement of urinary elimination, apart from the Nrf2 nuclear translocation, activation of Nrf2 regulation proteins, and promote detoxification of antioxidant enzymes (Gao et al., 2013).

\section{Concluding remarks and future perspectives}

The study of biological effects of natural matrices, including the discovering of their active constituents, is a hot topic that focuses the attention of numerous scientists. In fact, the use of botanical preparations dates since pre-historic era, but due to the lack of scientific evidences, including modes of action, no solid recognition or assertion was achieved. Prior to the use of synthetic drugs, 
botanical preparations were used for a multitude of health conditions, as also for averting the aging process. Then, with the increasing use of synthetic molecules, natural therapies passed to a second stage. But, currently, the focus on natural matrices (namely, rich in phenolic compounds) properties has gained attention. In vitro studies increased in an exponential manner, and numerous bioactive properties were confirmed; then, in vivo studies mainly in animal models have also increased, but in smaller proportions.

Despite those advances, to the majority of plant species no extensive knowledge is available, namely the responsible phytochemicals for the observed bioactivity, their mechanisms of action, therapeutic and prophylactic doses, synergism, antagonisms and other inter-relations between them. Clinical trials are very important, in order to develop future and effective alternatives to improve the health and well-being of individuals. In addition, it is of the major importance to prove the effective therapeutic potential of phytochemical preparations, together with the increasing number of publications related with the discovery of plant species with bioactive potential, as well as to evaluate other variables, such as bioavailability and bio-efficacy, nutrient-phytochemicals and drug-phytochemicals interactions.

\section{Acknowledgements}

The authors are grateful to Foundation for Science and Technology (FCT, Portugal) for N. Martins grant (SFRH/BD/87658/2012), L. Barros researcher contract under "Programa Compromisso com Ciência - 2008" and financial support to the research centre CIMO (strategic project Pest-OE/AGR/UI0690/2014).

\section{References}

Adefegha, S. A., \& Oboh, G. (2012). Effect of diets supplemented with Ethiopian pepper [Xylopia aethiopica (Dun.) A. Rich (Annonaceae)] and Ashanti pepper [Piper guineense Schumach. et Thonn (Piperaceae)] on some biochemical parameters in normal rats. Asian Pacific Journal of Tropical Biomedicine, 2 (2 Suppl.), S558-S566.

Ademiluyi, A. O., \& Oboh, G. (2012). Attenuation of oxidative stress and hepatic damage by some fermented tropical legume condiment diets in streptozotocininduced diabetes in rats. Asian Pacific Journal of Tropical Medicine, 5(9), 692-697.

Almaraz-Abarca, N., Campos, M. G., Ávila-Reyes, J. A., Naranjo-Jiménez, N., Corral, J. H., \& González-Valdez, L. S. (2007). Antioxidant activity of polyphenolic extract of monofloral honeybee-collected pollen from mesquite (Prosopis juliflora, Leguminosae). Journal of Food Composition and Analysis, 20(2), 119-124.

Aslan, M., Orhan, D. D., Orhan, N., Sezik, E., \& Yesilada, E. (2007). In vivo antidiabetic and antioxidant potential of Helichrysum plicatum ssp. plicatum capitulums in streptozotocin-induced-diabetic rats. Journal of Ethnopharmacology, 109(1), 54-59.

Atawodi, S. E., Iliemene, D. U., \& Onyike, E. (2014). In vivo antioxidant effect of methanolic extract of Afzelia africana seed on carbon tetrachloride-induced acute and chronic oxidative injury in rats. International Journal of Agriculture \& Biology, 16(3), 597-602.

Avello, M. A., Pastene, E. R., Bustos, E. D., Bittner, M. L., \& Becerra, J. A. (2013). Variation in phenolic compounds of Ugni molinae populations and their potential use as antioxidant supplement. Brazilian Journal of Pharmacognosy, 23(1), 44-50.

Bebrevska, L., Foubert, K., Hermans, N., Chatterjee, S., Marck, E. Van, Meyer, G., et al. (2010). In vivo antioxidative activity of a quantified Pueraria lobata root extract. Journal of Ethnopharmacology, 127(1), 112-117.

Carocho, M., \& Ferreira, I. C. F. R. (2013). A review on antioxidants, prooxidants and related controversy: natural and synthetic compounds, screening and analysis methodologies and future perspectives. Food and Chemical Toxicology, 51, $15-25$.

Chang, W.-H., Chen, C.-M., Hu, S.-P., Kan, N.-W., Chiu, C.-C., \& Liu, J.-F. (2007). Effect of purple sweet potato leaves consumption on the modulation of the immune response in basketball players during the training period. Asia Pacific Journal of Clinical Nutrition, 16(4), 609-615.

Chaturvedi, R. K., \& Beal, M. F. (2013). Mitochondrial diseases of the brain. Free Radical Biology \& Medicine, 63, 1-29.

Cheng, N., Ren, N., Gao, H., Lei, X., Zheng, J., \& Cao, W. (2013). Antioxidant and hepatoprotective effects of Schisandra chinensis pollen extract on CCl4-induced acute liver damage in mice. Food and Chemical Toxicology, 55, 234-240.

Chen, C.-M., Lin, Y.-L., Chen, C.-Y. O., Hsu, C.-Y., Shieh, M.-J., \& Liu, J.-F. (2008), Consumption of purple sweet potato leaves decreases lipid peroxidation and
DNA damage in humans. Asia Pacific Journal of Clinical Nutrition, 17(3), 408-414. Contini, M., Baccelloni, S., Frangipane, M. T., Merendino, N., \& Massantini, R. (2012) Increasing espresso coffee brew antioxidant capacity using phenolic extrac recovered from hazelnut skin waste. Journal of Functional Foods, 4(1), 137-146. Cueva, C., Moreno-Arribas, M. V., Martín-Álvarez, P. J., Bills, G., Vicente, M. E. Basilio, A., et al. (2010). Antimicrobial activity of phenolic acids against commensal, probiotic and pathogenic bacteria. Research in Microbiology, 161 $372-382$.

Dai, J., \& Mumper, R. J. (2010). Plant phenolics: extraction, analysis and their antioxidant and anticancer properties. Molecules, 15(10), 7313-7352.

Dehghan, G., Shafiee, A., Ghahremani, M. H., Ardestani, S. K., \& Abdollahi, M. (2007) Antioxidant potential of various extracts from Ferula szovitsiana in relation to their phenolic content. Pharmaceutical Biology, 45(9), 691-699.

Devasagayam, T. P. A., Tilak, J. C., Boloor, K. K., Sane, K. S., Ghaskadbi, S. S., \& Lele, R. D. (2004). Free radicals and antioxidants in human health: current status and future prospects. Journal of the Association of Physicians of India, 52(October), 794-804.

Dey, T. B., \& Kuhad, R. C. (2014). Enhanced production and extraction of phenolic compounds from wheat by solid-state fermentation with Rhizopus oryzae RCK2012. Biotechnology Reports, 4, 120-127.

Duarte-Almeida, J. M., Novoa, A. V., Linares, A. F., Lajolo, F. M., \& Genovese, M. I (2006). Antioxidant activity of phenolics compounds from sugar cane (Saccharum officinarum L.) juice. Plant Foods for Human Nutrition, 61(4), 187-192.

Ergin, V., Hariry, R. E., \& Karasu, C. (2013). Carbonyl stress in aging process: role of vitamins and phytochemicals as redox regulators. Aging and Disease, 4(5) $276-294$.

Espín, J. C., García-Conesa, M. T., \& Tomás-Barberán, F. A. (2007). Nutraceuticals: facts and fiction. Phytochemistry, 68(22-24), 2986-3008.

Fardet, A., Rock, E., \& Rémésy, C. (2008). Is the in vitro antioxidant potential of whole-grain cereals and cereal products well reflected in vivo? Journal of Cereal Science, 48(2), 258-276.

Farhat, M. B., Landoulsi, A., Chaouch-Hamada, R., Sotomayor, J. A., \& Jordán, M. J. (2013). Characterization and quantification of phenolic compounds and antioxidant properties of Salvia species growing in different habitats. Industrial Crops and Products, 49, 904-914.

Fernandez-Panchon, M. S., Villano, D., Troncoso, A. M., \& Garcia-Parrilla, M. C. (2008). Antioxidant activity of phenolic compounds: from in vitro results to in vivo evidence. Critical Reviews in Food Science and Nutrition, 48(7), 649-671.

Fetoni, A. R., Mancuso, C., Eramo, S. L. M., Ralli, M., Piacentini, R., Barone, E., et al (2010). In vivo protective effect of ferulic acid against noise-induced hearing loss in the guinea-pig. Neuroscience, 169(4), 1575-1588.

Gao, S., Duan, X., Wang, X., Dong, D., Liu, D., Li, X., et al. (2013). Curcumin attenuates arsenic-induced hepatic injuries and oxidative stress in experimental mice through activation of Nrf2 pathway, promotion of arsenic methylation and urinary excretion. Food and Chemical Toxicology, 59, 739-747.

Germanò, M. P., D'Angelo, V., Biasini, T., Sanogo, R., Pasquale, R., \& Catania, S. (2006) Evaluation of the antioxidant properties and bioavailability of free and bound phenolic acids from Trichilia emetica Vahl. Journal of Ethnopharmacology, 105(3), 368-373.

Gonçalves, A. E. S. S., Lellis-Santos, C., Curi, R., Lajolo, F. M., \& Genovese, M. I. (2014). Frozen pulp extracts of camu-camu (Myrciaria dubia McVaugh) attenuate the hyperlipidemia and lipid peroxidation of Type 1 diabetic rats. Food Research International, 64, 1-8.

Goodman, M., Bostick, R. M., Kucuk, O., \& Jones, D. P. (2011). Clinical trials of antioxidants as cancer prevention agents: past, present, and future. Free Radical Biology \& Medicine, 51(5), 1068-1084.

Gouthamchandra, K., Mahmood, R., \& Manjunatha, H. (2010). Free radical scavenging, antioxidant enzymes and wound healing activities of leaves extracts from Clerodendrum infortunatum L. Environmental Toxicology and Pharmacology, 30(1), 11-18.

Gris, E. F., Mattivi, F., Ferreira, E. A., Vrhovsek, U., Filho, D. W., Pedrosa, R. C., et al. (2013). Phenolic profile and effect of regular consumption of Brazilian red wines on in vivo antioxidant activity. Journal of Food Composition and Analysis, 31(1), $31-40$.

Grotewold, E. (2006). In Springer (Ed.), The science of flavonoids. Ohio, USA: United States of America: The Ohio State University - Colombus.

Halberstein, R. A. (2005). Medicinal plants: historical and cross-cultural usage patterns. Annals of Epidemiology, 15(9), 686-699.

Halliwell, B. (2012). Free radicals and antioxidants: updating a personal view. Nutrition Reviews, 70(5), 257-265.

Hamauzu, Y., Inno, T., Kume, C., Irie, M., \& Hiramatsu, K. (2006). Antioxidant and antiulcerative properties of phenolics from Chinese quince, quince, and apple fruits. Journal of Agricultural and Food Chemistry, 54(3), 765-772.

Hegde, P. S., Rajasekaran, N. S., \& Chandra, T. S. (2005). Effects of the antioxidant properties of millet species on oxidative stress and glycemic status in alloxaninduced rats. Nutrition Research, 25(12), 1109-1120.

Heim, K. E., Tagliaferro, A. R., \& Bobilya, D. J. (2002). Flavonoid antioxidants: Chemistry, metabolism and structure-activity relationships. Journal of Nutritional Biochemistry, 13(10), 572-584.

Hof, K. H. V. H., Kivits, G. A. A., Weststrate, J. A., \& Tijburg, L. B. M. (1998). Bioavailability of catechins from tea: the effect of milk. European Journal of Clinical Nutrition, 52(5), 356-359.

Hollman, P. C., Van Het, H. K. H., Tijburg, L. B. M., \& Katan, M. B. (2001). Addition of milk does not affect the absorption of flavonols from tea in man. Free Radical Research, 34(3), 297-300. 
Holst, B., \& Williamson, G. (2008). Nutrients and phytochemicals: from bioavailability to bioefficacy beyond antioxidants. Current Opinion in Biotechnology, 19(2), 73-82.

Hsouna, A. B., Saoudi, M., Trigui, M., Jamoussi, K., Boudawara, T., Jaoua, S., et al. (2011). Characterization of bioactive compounds and ameliorative effects of Ceratonia siliqua leaf extract against $\mathrm{CCl} 4$ induced hepatic oxidative damage and renal failure in rats. Food and Chemical Toxicology, 49(12), 3183-3191.

Huang, B., Ban, X., He, J., Tong, J., Tian, J., \& Wang, Y. (2010). Hepatoprotective and antioxidant activity of ethanolic extracts of edible lotus (Nelumbo nucifer Gaertn.) leaves. Food Chemistry, 120(3), 873-878.

Jaiswal, D., Rai, P. K., Mehta, S., Chatterji, S., Shukla, S., Rai, D. K., et al. (2013). Role of Moringa oleifera in regulation of diabetes-induced oxidative stress. Asian Pacific Journal of Tropical Medicine, 6(6), 426-432.

Kappel, V. D., Costa, G. M., Scola, G., Silva, F. A., Landell, M. F., Valente, P., et al. (2008). Phenolic content and antioxidant and antimicrobial properties of fruits of Capsicum baccatum L. var. pendulum at different maturity stages. Journal of Medicinal Food, 11(2), 267-274.

Kapravelou, G., Martínez, R., Andrade, A. M., Chaves, C. L., López-Jurado, M., Aranda, P., et al. (2015). Improvement of the antioxidant and hypolipidaemic effects of cowpea flours (Vigna unguiculata) by fermentation: results of in vitro and in vivo experiments. Journal of the Science of Food and Agriculture, 95(6), 1207-1216.

Kaushik, G., Satya, S., Khandelwal, R. K., \& Naik, S. N. (2010). Commonly consumed Indian plant food materials in the management of diabetes mellitus. Diabetes \& Metabolic Syndrome: Clinical Research \& Reviews, 4(1), 21-40.

Kay, C. D., \& Holub, B. J. (2002). The effect of wild blueberry (Vaccinium angustifo(ium) consumption on postprandial serum antioxidant status in human subjects. The British Journal of Nutrition, 88(4), 389-398.

Kountouri, A. M., Mylona, A., Kaliora, A. C., \& Andrikopoulos, N. K. (2007) Bioavailability of the phenolic compounds of the fruits (drupes) of Olea europaea (olives): Impact on plasma antioxidant status in humans. Phytomedicine, 14(10), 659-667.

Langley-Evans, S. C. (2000a). Antioxidant potential of green and black tea determined using the ferric reducing power (FRAP) assay. International Journal of Food Sciences and Nutrition, 51(3), 181-188.

Langley-Evans, S. C. (2000b). Consumption of black tea elicits an increase in plasma antioxidant potential in humans. International Journal of Food Sciences and Nutrition, 51(5), 309-315.

Larrosa, M., García-Conesa, M. T., Espín, J. C., \& Tomás-Barberán, F. A. (2010). Ellagitannins, ellagic acid and vascular health. Molecular Aspects of Medicine, 31(6), $513-539$.

Leenen, R., Roodenburg, A. J. C., Tijburg, L. B. M., \& Wiseman, S. A. (2000). A single dose of tea with or without milk increases plasma antioxidant activity in humans. European Journal of Clinical Nutrition, 54(1), 87-92.

Li, P., Jia, J., Zhang, D., Xie, J., Xu, X., \& Wei, D. (2013). In vitro and in vivo antioxidant activities of a flavonoid isolated from celery (Apium graveolens L. var. dulce) Food \& Function, 5, 50-56.

Li, A., Li, S., Zhang, Y., Xu, X., Chen, Y., \& Li, H. (2014). Resources and biological activities of natural polyphenols. Nutrients, 6, 6020-6047.

Lim, D. H., Choi, D., Choi, O.-Y., Cho, K.-A., Kim, R., Choi, H.-S., et al. (2011). Effect of Astragalus sinicus L. seed extract on antioxidant activity. Journal of Industrial and Engineering Chemistry, 17(3), 510-516.

Lorenz, M., Jochmann, N., Von Krosigk, A., Martus, P., Baumann, G., Stangl, K., et al. (2007). Addition of milk prevents vascular protective effects of tea. European Heart Journal, 28(2), 219-223.

Maheep, B., Sunil, V., Yogesh, V., Durgesh, S., \& Kanika, S. (2010). Antioxidant activity of fruit pulp powder of Cassia fistula. Pharmacognosy Journal, 2(8), 219-228.

Mazza, G., Kay, C. D., Cottrell, T., \& Holub, B. J. (2002). Absorption of anthocyanins from blueberries and serum antioxidant status in human subjects. Journal of Agricultural and Food Chemistry, 50, 7731-7737.

Molan, A. L., Lila, M. A., \& Mawson, J. (2008). Satiety in rats following blueberry extract consumption induced by appetite-suppressing mechanisms unrelated to in vitro or in vivo antioxidant capacity. Food Chemistry, 107(3), 1039-1044.

Mukherjee, P. K., \& Houghton, P. J. (2009). Evaluation of herbal medicinal products: Perspectives on quality, safety and efficacy. Great Britain: Royal Pharmaceutical Society of Great Britain.

Murray, M. T., \& Pizzorno, J. (2005). The Encyclopedia of healing foods. New York, NY: Atria Book.

Murray, M. T., \& Pizzorno, J. (2012). The encyclopedia of natural medicine. New York, NY: Atria Books.

Musumeci, G., Szychlinska, M. A., \& Mobasheri, A. (2015). Age-related degeneration of articular cartilage in the pathogenesis of osteoarthritis: molecular markers of senescent chondrocytes. Histology and Histopathology, 30(1), 1-12.

Naghdi Badi, H., Yazdani, D., Ali, S. M., \& Nazari, F. (2004). Effects of spacing and harvesting time on herbage yield and quality/quantity of oil in thyme, Thymus vulgaris L. Industrial Crops and Products, 19(3), 231-236.

Ndhlala, A. R., Stafford, G. I., \& Van Staden, J. (2013). Phytosynergy in some Hypoxis species and pharmacological properties of a Hypoxis-based phytopharmaceutical formula. Journal of Ethnopharmacology, 150(2), 492-500.

Parker, T. L., Wang, X. H., Pazmiño, J., \& Engeseth, N. J. (2007). Antioxidant capacity and phenolic content of grapes, sun-dried raisins, and golden raisins and thei effect on ex vivo serum antioxidant capacity. Journal of Agricultural and Food Chemistry, 55(21), 8472-8477.

Pinelo, M., Landbo, A.-K. R., Vikbjerg, A. F., \& Meyer, A. S. (2006). Effect of clarification techniques and rat intestinal extract incubation on phenolic composition and antioxidant activity of black currant juice. Journal of Agricultural and Food Chemistry, 54(18), 6564-6571.

Qingming, Y., Xianhui, P., Weibao, K., Hong, Y., Yidan, S., Li, Z., et al. (2010). Antioxidant activities of malt extract from barley (Hordeum vulgare L.) toward various oxidative stress in vitro and in vivo. Food Chemistry, 118(1), 84-89.

Que, F., Mao, L., \& Zheng, X. (2007). In vitro and vivo antioxidant activities of daylily flowers and the involvement of phenolic compounds. Asia Pacific Journal of Clinical Nutrition, 16(Suppl.1), 196-203.

Rai, S., Wahile, A., Mukherjee, K., Saha, B. P., \& Mukherjee, P. K. (2006). Antioxidant activity of Nelumbo nucifera (sacred lotus) seeds. Journal of Ethnopharmacology, 104(3), 322-327.

Ramirez-Tortosa, C., Andersen, Ø. M., Gardner, P. T., Morrice, P. C., Wood, S. G., Duthie, S. J., et al. (2001). Anthocyanin-rich extract decreases indices of lipid peroxidation and DNA damage in vitamin E-depleted rats. Free Radical Biology and Medicine, 31(9), 1033-1037.

Rein, D., Lotito, S., Holt, R. R., Keen, C. L., Schmitz, H. H., \& Fraga, C. G. (2000) Epicatechin in human plasma: in vivo determination and effect of chocolate consumption on plasma oxidation status. The Journal of Nutrition, 130(8S Suppl), 2109S-2114S

Rein, M. J., Renouf, M. Cruz-Hernandez, C. Actis-Goretta, L., Thakkar, S. K., \& Pinto, M. S. (2013). Bioavailability of bioactive food compounds: a challenging journey to bioefficacy. British Journal of Clinical Pharmacology, 75(3), 588-602.

Roche, H., \& Bogé, G. (2000). In vivo effects of phenolic compounds on blood parameters of a marine fish (Dicentrarchus labrax). Comparative Biochemistry and Physiology Part C, 125(3), 345-353.

Rubió, L., Motilva, M.-J., \& Romero, M.-P. (2013). Recent advances in biologically active compounds in herbs and spices: a review of the most effective antioxidant and anti-inflammatory active principles. Critical Reviews in Food Science and Nutrition, 53(9), 943-953.

Saeed, N., Khan, M. R., \& Shabbir, M. (2012). Antioxidant activity, total phenolic and total flavonoid contents of whole plant extracts Torilis leptophylla L. BMC Complementary and Alternative Medicine, 12(221), 1-12.

Samson, J., Sheeladevi, R., \& Ravindran, R. (2007). Oxidative stress in brain and antioxidant activity of Ocimum sanctum in noise exposure. NeuroToxicology, 28(3), 679-685.

Saura-Calixto, F., Serrano, J., \& Goñi, I. (2007). Intake and bioaccessibility of total polyphenols in a whole diet. Food Chemistry, 101(2), 492-501.

Scalbert, A., Déprez, S., Mila, I., Albrecht, A.-M., Huneau, J.-F., \& Rabot, S. (2000). Proanthocyanidins and human health: systemic effects and local effects in the gut. BioFactors, 13(1-4), 115-120.

Scholz, S., \& Williamson, G. (2007). Interactions affecting the bioavailability of dietary polyphenols in vivo. International Journal for Vitamin and Nutrition Research, 77(3), 224-235.

Sen, S., Chakraborty, R., Verma, A., De, B., Devanna, N., \& Dey, B. K. (2014). Evaluation of antioxidant activity of Saraca indica leaves extracts using in vitro and ex vivo models. Journal of Scientific and Industrial Research, 73(3), 157-162.

Sen, S., De, B., Devanna, N., \& Chakraborty, R. (2013). Total phenolic, total flavonoid content, and antioxidant capacity of the leaves of Meyna spinosa Roxb., an Indian medicinal plant. Chinese Journal of Natural Medicines, 11(2), 149-157.

Serafini, M., Bugianesi, R., Maiani, G., Valtuena, S., Santis, S., \& Crozier, A. (2003). Plasma antioxidants from chocolate. Nature, 424(6952), 1013.

Serafini, M., Bugianesi, R., Salucci, M., Azzini, E., Raguzzini, A., \& Maiani, G. (2002). Effect of acute ingestion of fresh and stored lettuce (Lactuca sativa) on plasma total antioxidant capacity and antioxidant levels in human subjects. The British Journal of Nutrition, 88(6), 615-623.

Serafini, M., Laranjinha, J. A. N., Almeida, L. M., \& Maiani, G. (2000). Inhibition of human LDL lipid peroxidation by phenol-rich beverages and their impact on plasma total antioxidant capacity in humans. Journal of Nutritional Biochemistry, 11(11-12), 585-590.

Serafini, M., Testa, M. F., Villaño, D., Pecorari, M., Wieren, K. V., Azzini, E., et al. (2009). Antioxidant activity of blueberry fruit is impaired by association with milk. Free Radical Biology and Medicine, 46(6), 769-774.

Shi, G.-F., An, L.-J., Jiang, B., Guan, S., \& Bao, Y.-M. (2006). Alpinia protocatechuic acid protects against oxidative damage in vitro and reduces oxidative stress in vivo. Neuroscience Letters, 403(3), 206-210.

Silva, J. K., Cazarin, C. B. B., Colomeu, T. C., Batista, Â. G., Meletti, L. M. M. Paschoal, J. A. R., et al. (2013). Antioxidant activity of aqueous extract of passion fruit (Passiflora edulis) leaves: In vitro and in vivo study. Food Research International, 53(2), 882-890.

Singh, R. P., Sharad, S., \& Kapur, S. (2004). Free radicals and oxidative stress in neurodegenerative diseases: relevance of dietary antioxidants. Journal Indian Academy of Clinical Medicine, 5(3), 218-225.

Siqueira, E. M. A., Marin, A. M. F., Cunha, M. D. S. B., Fustinoni, A. M., Sant'Ana, L. P., \& Arruda, S. F. (2012). Consumption of baru seeds [Dipteryx alata Vog.], a Brazilian savanna nut, prevents iron-induced oxidative stress in rats. Food Research International, 45(1), 427-433.

Somanah, J., Bourdon, E., Rondeau, P., Bahorun, T., \& Aruoma, O. I. (2014). Relationship between fermented papaya preparation supplementation, erythrocyte integrity and antioxidant status in pre-diabetics. Food and Chemical Toxicology, $65,12-17$.

Sulaiman, S. F., Sajak, A. A. B., Ooi, K. L., \& Seow, E. M. (2011). Effect of solvents in extracting polyphenols and antioxidants of selected raw vegetables. Journal of Food Composition and Analysis, 24(4-5), 506-515.

Sung, H., Nah, J., Chun, S., Park, H., Yang, S. E., \& Min, W. K. (2000). In vivo antioxidant effect of green tea. European Journal of Clinical Nutrition, 54(7), 527-529. 
Sunil, C., \& Ignacimuthu, S. (2011). In vitro and in vivo antioxidant activity of Symplocos cochinchinensis S. Moore leaves containing phenolic compounds. Food and Chemical Toxicology, 49(7), 1604-1609.

Su, X.-Y., Wang, Z.-Y., \& Liu, J.-R. (2009). In vitro and in vivo antioxidant activity of Pinus koraiensis seed extract containing phenolic compounds. Food Chemistry, 117(4), 681-686.

Taârit, M. B., Msaada, K., Hosni, K., \& Marzouk, B. (2012). Physiological changes, phenolic content and antioxidant activity of Salvia officinalis L. grown under saline conditions. Journal of the Science of Food and Agriculture, 92(8), 1614-1619.

Trabelsi, N., Waffo-Téguo, P., Snoussi, M., Ksouri, R., Mérillon, J. M., Smaoui, A., et al. (2012). Variability of phenolic composition and biological activities of two Tunisian halophyte species from contrasted regions. Acta Physiologiae Plantarum, 35(3), 749-761.

Tulipani, S., Alvarez-Suarez, J. M., Busco, F., Bompadre, S., Quiles, J. L., Mezzetti, B., et al. (2011). Strawberry consumption improves plasma antioxidant status and erythrocyte resistance to oxidative haemolysis in humans. Food Chemistry, $128(1), 180-186$.

Valko, M., Leibfritz, D., Moncol, J., Cronin, M. T. D., Mazur, M., \& Telser, J. (2007). Free radicals and antioxidants in normal physiological functions and human disease. The International Journal of Biochemistry \& Cell Biology, 39(1), 44-84.

Vanaclocha, B., \& Cañigueral, S. (2003). In Masson (Ed.), Fitoterapia: Vademecum de Prescripción (4th ed.) (Barcelona).

Velderrain-Rodríguez, G. R., Palafox-Carlos, H., Wall-Medrano, A., Ayala-Zavala, J. F., Chen, C.-Y. O., Robles-Sánchez, M., et al. (2014). Phenolic compounds: their journey after intake. Food \& Function, 5(2), 189-197.

Verma, A. R., Vijayakumar, M., Mathela, C. S., \& Rao, C. V. (2009). In vitro and in vivo antioxidant properties of different fractions of Moringa oleifera leaves. Food and Chemical Toxicology, 47(9), 2196-2201.

Verma, A. R., Vijayakumar, M., Rao, C. V., \& Mathela, C. S. (2010). In vitro and in vivo antioxidant properties and DNA damage protective activity of green fruit of Ficus glomerata. Food and Chemical Toxicology, 48(2), 704-709.

Vulić, J. J., Ćebović, T. N., Ćanadanović-Brunet, J. M., Ćetković, G. S., Canadanović, V. M., Djilas, S. M., et al. (2014). In vivo and in vitro antioxidant effects of beetroot pomace extracts. Journal of Functional Foods, 6(1), 168-175.
Wang, H., Gao, X. D., Zhou, G. C., Cai, L., \& Yao, W. B. (2008). In vitro and in vivo antioxidant activity of aqueous extract from Choerospondias axillaris fruit. Food Chemistry, 106(3), 888-895.

Wang, W., \& Goodman, M. T. (1999). Antioxidant property of dietary phenolic agents in a human LDL-oxidation ex vivo model: interaction of protein binding activity. Nutrition Research, 19(2), 191-202.

Wang, X., Yang, L., Yang, X., \& Tian, Y. (2014). In vitro and in vivo antioxidant and antimutagenic activities of polyphenols extracted from hops (Humulus lupulus L.). Journal of the Science of Food and Agriculture, 94(8), 1693-1700.

Wojdylo, A., Oszmianski, J., \& Czemerys, R. (2007). Antioxidant activity and phenolic compounds in 32 selected herbs. Food Chemistry, 105(3), 940-949.

Wu, C. Y., Chen, R., Wang, X. S., Shen, B., Yue, W., \& Wu, Q. (2013). Antioxidant and anti-fatigue activities of phenolic extract from the seed coat of Euryale ferox Salisb. and identification of three phenolic compounds by LC-ESI-MS/MS. Molecules, 18(9), 11003-11021.

Xie, Y, Zhao, O Y Li, H. Y, Zhou, X, Liu, Y \& Zhang H (2014). Curcumin ameliorates cognitive deficits heavy ion irradiation-induced learning and memory deficits through enhancing of Nrf2 antioxidant signaling pathways. Pharmacology, Biochemistry, and Behavior, 126, 181-186.

Yeh, C.-T. \& Yen, G.-C. (2006). Modulation of hepatic phase II phenol sulfotransferase and antioxidant status by phenolic acids in rats. Journal of Nutritional Biochemistry, 17(8), 561-569.

Zhao, Z., Egashira, Y., \& Sanada, H. (2005). Phenolic antioxidants richly contained in corn bran are slightly bioavailable in rats. Journal of Agricultural and Food Chemistry, 53(12), 5030-5035.

Zhao, Z., Xu, Z., Le, K., Azordegan, N., Riediger, N. D., \& Moghadasian, M. H. (2009). Lack of evidence for antiatherogenic effects of wheat bran or corn bran in apolipoprotein E-knockout mice. Journal of Agricultural and Food Chemistry, 57(14), 6455-6460.

Zhao, J. G., Yan, Q.-Q., Lu, L.-Z., \& Zhang, Y.-Q. (2013). In vivo antioxidant, hypoglycemic, and anti-tumor activities of anthocyanin extracts from purple sweet potato. Nutrition Research and Practice, 7(5), 359-365.

Zhao, H., Zhang, H., \& Yang, S. (2014). Phenolic compounds and its antioxidant activities in ethanolic extracts from seven cultivars of Chinese jujube. Food Science and Human Wellness, 3(3-4), 183-190. 